\title{
Parental awareness regarding pediatric antibiotic use in Madinah, Saudi Arabia
}

\author{
Hani MJ Khojah ${ }^{1 *}$, Hosam G Abdelhady ${ }^{1}$, Abdulmalik A Alqurashi ${ }^{1}$, Enas M \\ Kasem $^{2}$, Nuha S Osailan', Mohammed O Alnuman', Ryan E Alruhaili ${ }^{2}$, \\ Abdulrahman A Bedaiwy ${ }^{2}$, Razan H Mofti ${ }^{1}$, Hassan A Abdel-Salam ${ }^{1,3}$ \\ ${ }^{1}$ College of Pharmacy, ${ }^{2}$ College of Medicine, Taibah University, Madinah, Saudi Arabia; ${ }^{3}$ Department of Microbiology, Faculty of \\ Pharmacy, Zagazig University, Zagazig, Egypt
}

*For correspondence: Email: hkhojah@taibahu.edu.sa; Tel: +966 505232584

Sent for review: 5 June 2019

Revised accepted: 25 January 2020

\begin{abstract}
Purpose: To determine the level of awareness of antibiotic use in children in Madinah, Saudi Arabia, and to identify factors associated with parental decisions regarding it.

Methods: Using a multiple-choice-question-based questionnaire survey, 1256 forms were distributed to visitors of major shopping malls in Madinah City to obtain socio-demographic and antibiotics knowledgebased data from October 2017 to January 2018. Differences in scores between and within groups on knowledge of parents about antibiotics were determined.

Results: Most participants (67\%) had good basic knowledge of antibiotics: 69 and $40 \%$ of respondents were aware of their side effects and antibacterial resistance, respectively. Participants in high age groups (> 46 years old) have a significantly higher mean knowledge score $(55.4 \pm 20.1, p<0.05)$ than those in younger groups. Educational status increased the mean knowledge score by approximately 60 $\%$, with the most educated group having a mean score of $61.2 \pm 16.4(p<0.05)$.

Conclusion: These results reveal the importance of awareness campaigns on antibiotic use and the role of healthcare professionals in the education of patients and parents on correct use of antibiotics, as well as the significance of antibacterial resistance.
\end{abstract}

Keywords: Antibiotics misuse, Pediatrics, Patient education, Antibacterial resistance

This is an Open Access article that uses a fund-ing model which does not charge readers or their institutions for access and distributed under the terms of the Creative Commons Attribution License (http://creativecommons.org/licenses/by/4.0) and the Budapest Open Access Initiative (http://www.budapestopenaccessinitiative.org/read), which permit unrestricted use, distribution, and reproduction in any medium, provided the original work is properly credited.

Tropical Journal of Pharmaceutical Research is indexed by Science Citation Index (SciSearch), Scopus, International Pharmaceutical Abstract, Chemical Abstracts, Embase, Index Copernicus, EBSCO, African Index Medicus, JournalSeek, Journal Citation Reports/Science Edition, Directory of Open Access Journals (DOAJ), African Journal Online, Bioline International, Open-J-Gate and Pharmacy Abstracts

\section{INTRODUCTION}

Antibiotics have played vital roles in the treatment and prevention of various infectious diseases since the discovery of the first antibiotic (penicillin) in the 1940s. However, the misuse of antibiotics has led to side effects that sometimes become life-threatening. Thus, appropriate use of antibiotics is necessary in order to obtain their benefits with minimal side effects. However, in developing countries, there is rampant and persistent use of non-prescribed antibiotics among many individuals [1-5]. This improper behavior plays a major role in the emergence of antimicrobial resistance.

Antimicrobial resistance has resulted in serious adverse effects, particularly in children. 
Therefore, there is need for emphasis on parental education regarding the appropriate use of antibiotics [6]. This has been the focus of a large number of studies worldwide, including studies in Greece (Europe) [7], Enugu in Nigeria [8], Vietnam (Asia) [9], China [10], Navi Mumbai (India) [11], Nablus city in Palestine [12], as well as Riyadh and central region in Saudi Arabia [13, 14]. The control of use of antibiotics is a primary step in the management of antimicrobial resistance and related adverse effects.

Antibiotics alter gastrointestinal microbiota [15]. A link between the gut microbiota and brain function has shed more light on the pathophysiology of several psychiatric disorders such as depression and Alzheimer's disease [16]. The importance of the gut microbiota in the development of the cardiovascular and nervous systems has also been established [16, 17]. Thus, awareness of proper use of antibiotic may help to avoid the development of resistant strains of bacteria, while eradicating diseases among the young.

So far, this is the first study conducted in Madinah, Saudi Arabia, to evaluate the awareness of parents and guardians on the use of antibiotics for children. Using a scoring system, this study was carried out to assess general knowledge of parents on antibiotics, with respect to adverse effects following repeated administration of oral, non-prescribed antibiotics to children. The likely factors associated with parental decisions regarding antibiotic use for children were also measured.

\section{METHODS}

This multiple-choice-question-based questionnaire survey was conducted between October, 2017 and January, 2018 in Madinah. The questionnaires were distributed to visitors at health campaign points in major shopping malls in Madinah City.

\section{Ethical approval}

The study was approved by the Research Ethics Committee of the College of Pharmacy, Taibah University, Saudi Arabia (approval no. UCPREC/20180826/Khojah), and was conducted in accordance with the ethical guidelines of the Declaration of Helsinki [18]. Confidentiality and privacy of participants' data were ensured through anonymous questionnaire distribution. All participants consented to having their information used in this study.

\section{Questionnaire design}

The multiple-choice-question-based questionnaire survey was designed to allow for an accurate evaluation of parental awareness, understanding, and practice of pediatric antibiotic misuse. The first section of the questionnaire was designed to collect participants' general information, including socio-demographic data such as age, sex, number of children, and level of education. Participants were then asked to evaluate their level of general knowledge on antibiotics, the ideal use and mechanism of action, and how important they believed educating parents on antibiotics was. Parents who admitted to obtaining antibiotics unlawfully for their children, i.e. without a doctor's prescription, were asked further questions to allow for a better understanding of the reasons behind their actions. Moreover, their compliance with medication instructions was evaluated.

Participants were then presented with a scorebased test covering three main areas: antibioticsrelated general knowledge, side effects of antibiotics use, and awareness of, and prevention of bacterial resistance.

\section{Statistical analysis}

Data are presented as frequencies, percentages, or mean \pm standard deviation (SD) as appropriate. Independent t-test and one-way analysis of variance (ANOVA) were used to investigate percentage differences in scores between and within groups. Data were statistically analyzed using the $20^{\text {th }}$ version of the Statistical Package for the Social Sciences (SPSS, International Business Machines Corp., Armonk, New York, USA). Values of $p<0.5$ were considered significant.

\section{RESULTS}

A total of 1256 completed questionnaires were collected. Demographic data distribution (Table 1) showed that most participants were married, had children, and were aged above 25 years. More than half $(75.5 \%)$ of participants had received undergraduate courses at university level, while approximately $23 \%$ received school education or less. The majority of participants lived in Madinah city, while just over $8 \%$ lived in the surrounding suburb areas. Participants' level of general knowledge on antibiotics, their ideal use, and their mechanisms of action, as well as their views on the importance of healthcare education for parents, are presented on the basis of demographic grouping (Table 1). With the exception of the poorly educated participants, all 
demographic groups demonstrated intermediate level of knowledge. The number of participants claiming to have high level of knowledge on antibiotics increased with increasing levels of education. However, a surprisingly large percentage (28.6 \%) of the least educated group claimed to have had a high level of knowledge in all three areas. When participants were tested on all three areas, there was a significant, positive correlation between level of education and participants' score $(p<0.05)$, with the poorly educated groups scoring the lowest in all areas (Table 2).

In addition, age of participants seemed to have had a significant effect on the level of knowledge of participants, in all tested areas. Table 2 shows that the average score for participants was significantly increased with increasing age. Surprisingly, this correlation was less apparent with the number of children a parent had. This indicated that the number of children exerted little effect on the parents' awareness of antibiotic misuse. Results also showed that living in the suburb areas had a significant lowering effect on participants' average scores in all three tested areas (Table 2). In addition, much like all other groups, participants from the suburb areas strongly believed in the need to educate parents on the use of antibiotics.

The majority of parents who admitted to unlawful administration of antibiotics to their children did so when their children showed symptoms of general cold (39\%) or simply when they had high fever (43\%) (Table 3$)$. Overall, $61.8 \%$ of those parents claimed that they did not to buy antibiotics whenever their children were ill, indicating some level of self-restraint. Table 4 shows that the participants in this group had a higher average knowledge scores (in all three tested areas) than parents who bought antibiotics each time their children were sick (general knowledge score: 77.8 vs 61.2 ; side effects knowledge score: 59.2 vs 52.5, and antibacterial resistance knowledge score: 24.5 vs 24.1, respectively).

Nevertheless, more than $40 \%$ failed to follow the recommended dose regimen, and may even have stored the leftover doses for future use. Sadly, this could have had a great effect on antibiotic resistance for the whole population and could also have had a catastrophic effect on the children's health.

Table 3: Current practices among parents who gave antibiotics to their children without prescription

\begin{tabular}{lc}
\hline Practice $(n=400)$ & Frequency, $\boldsymbol{n}(\%)$ \\
\hline Buying antibiotics each time the child was sick & $153(38.3)$ \\
Yes & $247(61.8)$ \\
No & $174(43.5)$ \\
Reason for antibiotic use & $31(7.8)$ \\
Fever & $39(9.8)$ \\
Wound & $156(39.0)$ \\
Gastrointestinal symptoms & $104(26)$ \\
Cold & $82(20.5)$ \\
Discontinuation time & $214(53.5)$ \\
At no symptoms & \\
After full course of doses without proper timing & $90(22.5)$ \\
After full course of doses with proper timing & $310(77.5)$ \\
Keeping the remaining doses for further use & \\
Yes & \\
No & \\
\hline
\end{tabular}

Table 5: Reasons for purchasing antibiotics without a prescription

\begin{tabular}{lc}
\hline Reason (n=1256) & Frequency, $\mathbf{n}(\%)^{\star}$ \\
\hline Lack of knowledge on the importance of & $652(51.9)$ \\
consulting a physician & $760(60.5)$ \\
Long waiting time at clinics & $325(25.9)$ \\
Expenses involved in visiting private clinics & $304(24.2)$ \\
Availability of antibiotics information on the & \\
internet & $340(27.1)$ \\
Ease of obtaining antibiotics from retail & $463(36.9)$ \\
pharmacies without prescriptions & $185(14.7)$ \\
Previous experience with similar antibiotics & Advice from other antibiotic users
\end{tabular}

*Participants were allowed to give more than one reason for obtaining antibiotics without a legal prescription 
Table 1: Participants' demographic information and frequencies of the claimed level of general knowledge about antibiotics

\begin{tabular}{|c|c|c|c|c|c|c|c|c|c|c|c|c|c|}
\hline \multicolumn{3}{|c|}{$\begin{array}{l}\text { Demographic characteristics } \\
(n=1256)\end{array}$} & \multicolumn{4}{|c|}{$\begin{array}{l}\text { Level of the general knowledge on antibiotics and } \\
\text { their ideal use }\end{array}$} & \multicolumn{4}{|c|}{$\begin{array}{l}\text { Level of knowledge on antibiotics mechanism of } \\
\text { action }\end{array}$} & \multicolumn{3}{|c|}{$\begin{array}{l}\text { Opinion regarding the importance } \\
\text { of health education for parents }\end{array}$} \\
\hline & & & None & Weak & Intermediate & High & None & Weak & Intermediate & High & \begin{tabular}{|l|} 
Not \\
needed
\end{tabular} & $\begin{array}{l}\text { As } \\
\text { needed }\end{array}$ & Needed \\
\hline Variable & & $\begin{array}{l}\text { Frequency } \\
n(\%)\end{array}$ & $\begin{array}{l}\text { Frequency } \\
n(\%)\end{array}$ & $\begin{array}{l}\text { Frequency } \\
n(\%)\end{array}$ & $\begin{array}{l}\text { Frequency } \\
n(\%)\end{array}$ & $\begin{array}{l}\text { Frequency } \\
n(\%)\end{array}$ & $\begin{array}{l}\text { Frequency } \\
n(\%)\end{array}$ & $\begin{array}{l}\text { Frequency } \\
n(\%)\end{array}$ & $\begin{array}{l}\text { Frequency } \\
n(\%)\end{array}$ & $\begin{array}{l}\text { Frequency } \\
n(\%)\end{array}$ & $\begin{array}{l}\text { Frequency } \\
n(\%)\end{array}$ & $\begin{array}{l}\text { Frequency } \\
n(\%)\end{array}$ & $\begin{array}{l}\text { Frequency } \\
n(\%)\end{array}$ \\
\hline Sex & \begin{tabular}{|l} 
Male \\
Female
\end{tabular} & $\begin{array}{l}269(21.4) \\
987(78.6)\end{array}$ & $\begin{array}{l}12(4.5) \\
34(3.4)\end{array}$ & $\begin{array}{l}48(17.8) \\
145(14.7)\end{array}$ & $\begin{array}{l}171(63.6) \\
660(66.9)\end{array}$ & $\begin{array}{l}38(14.1) \\
148(15.0)\end{array}$ & $\begin{array}{l}20(7.4) \\
73(7.4)\end{array}$ & $\begin{array}{l}63(23.4) \\
192(19.5)\end{array}$ & $\begin{array}{l}149(55.4) \\
583(59.1)\end{array}$ & $\begin{array}{l}37(13.8) \\
139(14.1)\end{array}$ & $\begin{array}{l}4(1.5) \\
3(0.3)\end{array}$ & $\begin{array}{l}58(21.6) \\
169(17.1)\end{array}$ & $\begin{array}{l}207(77.0) \\
815(82.6)\end{array}$ \\
\hline $\begin{array}{l}\text { Marital } \\
\text { status }\end{array}$ & $\begin{array}{l}\text { Married } \\
\text { Unmarried }\end{array}$ & $\begin{array}{l}1129 \\
(89.9) \\
127(10.1)\end{array}$ & $\begin{array}{l}42(3.7) \\
4(3.1)\end{array}$ & $\begin{array}{l}166(14.7) \\
27(21.3)\end{array}$ & $\begin{array}{l}756(67.0) \\
75(59.1)\end{array}$ & $\begin{array}{l}165(14.6) \\
21(16.5)\end{array}$ & $\begin{array}{l}88(7.8) \\
5(3.9)\end{array}$ & $\begin{array}{l}235(20.8) \\
20(15.7)\end{array}$ & $\begin{array}{l}652(57.8) \\
80(63.0)\end{array}$ & $\begin{array}{l}154(13.6) \\
22(17.3)\end{array}$ & $\begin{array}{l}7(0.6) \\
0(0.0)\end{array}$ & $\begin{array}{l}206(18.2) \\
21(16.5)\end{array}$ & $\begin{array}{c}916(81.1) \\
106(83.5)\end{array}$ \\
\hline $\begin{array}{l}\text { Having } \\
\text { children }\end{array}$ & $\begin{array}{l}\text { No children } \\
\text { One child } \\
\text { More than a } \\
\text { child }\end{array}$ & $\begin{array}{l}198(15.8) \\
211(16.8) \\
847(67.4)\end{array}$ & $\begin{array}{l}10(5.1) \\
7(3.3) \\
29(3.4)\end{array}$ & $\begin{array}{l}36(18.2) \\
40(19.0) \\
117(13.8)\end{array}$ & $\begin{array}{l}121(61.1) \\
131(62.1) \\
579(68.4)\end{array}$ & $\begin{array}{l}31(15.7) \\
33(15.6) \\
122(14.4)\end{array}$ & $\begin{array}{l}10(5.1) \\
18(8.5) \\
65(7.7)\end{array}$ & $\begin{array}{l}39(19.7) \\
50(23.7) \\
166(19.6)\end{array}$ & $\begin{array}{l}119(60.1) \\
106(50.2) \\
507(59.9)\end{array}$ & $\begin{array}{l}30(15.2) \\
37(17.5) \\
109(12.9)\end{array}$ & $\begin{array}{l}0(0.0) \\
0(0.0) \\
7(0.8)\end{array}$ & $\begin{array}{l}29(14.6) \\
41(19.4) \\
157(18.5)\end{array}$ & $\begin{array}{l}169(85.4) \\
170(80.6) \\
683(80.6)\end{array}$ \\
\hline Age group & $\begin{array}{l}16-25 \\
26-35 \\
36-45 \\
>45\end{array}$ & $\begin{array}{l}247(19.7) \\
465(37.0) \\
349(27.8) \\
195(15.5)\end{array}$ & $\begin{array}{l}14(5.7) \\
12(2.6) \\
13(3.7) \\
7(3.6)\end{array}$ & $\begin{array}{l}52(21.1) \\
69(14.8) \\
56(16.0) \\
16(8.2)\end{array}$ & $\begin{array}{l}148(59.9) \\
316(68.0) \\
229(65.6) \\
138(70.8)\end{array}$ & $\begin{array}{l}33(13.4) \\
68(14.6) \\
51(14.6) \\
34(17.4)\end{array}$ & $\begin{array}{l}20(8.1) \\
33(7.1) \\
24(6.9) \\
16(8.2)\end{array}$ & $\begin{array}{l}56(22.7) \\
101(21.7) \\
74(21.2) \\
24(12.3)\end{array}$ & $\begin{array}{l}133(53.8) \\
267(57.4) \\
207(59.3) \\
125(64.1)\end{array}$ & $\begin{array}{l}38(15.4) \\
64(13.8) \\
44(12.6) \\
30(15.4)\end{array}$ & $\begin{array}{l}0(0.0) \\
5(1.1) \\
2(0.6) \\
0(0.0)\end{array}$ & $\begin{array}{l}51(20.6) \\
88(18.9) \\
61(17.5) \\
27(13.8)\end{array}$ & $\begin{array}{l}196(79.4) \\
372(80.0) \\
286(81.9) \\
168(86.2)\end{array}$ \\
\hline Residency & $\begin{array}{l}\text { Madinah City } \\
\text { Madinah } \\
\text { suburb }\end{array}$ & $\begin{array}{l}1154 \\
(91.9) \\
102(8.1)\end{array}$ & $\begin{array}{l}39(3.4) \\
7(6.9)\end{array}$ & $\begin{array}{l}178(15.4) \\
15(14.7)\end{array}$ & $\begin{array}{l}762(66.0) \\
69(67.7)\end{array}$ & $\begin{array}{l}175(15.2) \\
11(10.8)\end{array}$ & $\begin{array}{l}85(7.4) \\
8(7.8)\end{array}$ & $\begin{array}{l}234(20.3) \\
21(20.6)\end{array}$ & $\begin{array}{l}670(58.1) \\
62(60.8)\end{array}$ & $\begin{array}{l}165(14.3) \\
11(10.8)\end{array}$ & $\begin{array}{l}6(0.5) \\
1(1.0)\end{array}$ & $\begin{array}{l}210(18.2) \\
17(16.7)\end{array}$ & $\begin{array}{l}938(81.3) \\
84(82.4)\end{array}$ \\
\hline $\begin{array}{l}\text { Educational } \\
\text { level }\end{array}$ & $\begin{array}{l}\text { Uneducated } \\
\text { Elementary } \\
\text { Intermediate } \\
\text { Secondary } \\
\text { University } \\
\text { Postgraduate }\end{array}$ & $\begin{array}{l}7(0.6) \\
21(1.7) \\
51(4.1) \\
228(18.2) \\
862(68.6) \\
87(6.9)\end{array}$ & $\begin{array}{l}0(0.0) \\
4(19.0) \\
4(7.8) \\
15(6.6) \\
22(2.6) \\
1(1.1)\end{array}$ & $\begin{array}{l}3(42.9) \\
5(23.8) \\
10(19.6) \\
37(16.2) \\
132(15.3) \\
6(6.9)\end{array}$ & $\begin{array}{l}2(28.6) \\
11(52.4) \\
33(64.7) \\
143(62.7) \\
588(68.2) \\
54(62.1)\end{array}$ & $\begin{array}{l}2(28.6) \\
1(4.8) \\
4(7.8) \\
33(14.5) \\
120(13.9) \\
26(29.9)\end{array}$ & $\begin{array}{l}4(57.1) \\
9(42.9) \\
9(17.6) \\
21(9.2) \\
48(5.6) \\
2(2.3)\end{array}$ & $\begin{array}{l}1(14.3) \\
7(33.3) \\
12(23.5) \\
50(21.9) \\
175(20.3) \\
10(11.5)\end{array}$ & $\begin{array}{l}1(14.3) \\
4(19) \\
27(52.9) \\
127(55.7) \\
522(60.6) \\
51(58.6)\end{array}$ & $\begin{array}{l}1(14.3) \\
1(4.8) \\
3(5.9) \\
30(13.2) \\
117(13.6) \\
24(27.6)\end{array}$ & $\begin{array}{l}1(14.3) \\
0(0.0) \\
1(2.0) \\
2(0.9) \\
2(0.2) \\
1(1.1)\end{array}$ & $\begin{array}{l}1(14.3) \\
8(38.1) \\
18(35.3) \\
51(22.4) \\
135(15.7) \\
14(16.1)\end{array}$ & $\begin{array}{l}5(71.4) \\
13(61.9) \\
32(62.7) \\
175(76.8) \\
725(84.1) \\
72(82.8)\end{array}$ \\
\hline
\end{tabular}


Table 2: Level of significance of the differences in the means of various scores between various characteristics

\begin{tabular}{|c|c|c|c|c|c|c|c|c|c|}
\hline \multirow{2}{*}{\multicolumn{2}{|c|}{$\begin{array}{l}\text { Variables } \\
(n=1256)\end{array}$}} & \multirow{2}{*}{\multicolumn{2}{|c|}{$\begin{array}{l}\text { General knowledge score (out } \\
\text { of } 100 \text { ) }\end{array}$}} & \multirow{2}{*}{\multicolumn{2}{|c|}{$\begin{array}{l}\text { Side effects knowledge score } \\
\text { (out of } 100 \text { ) }\end{array}$}} & \multirow{2}{*}{\multicolumn{2}{|c|}{$\begin{array}{l}\text { Antibacterial resistance } \\
\text { knowledge score (out of 100) }\end{array}$}} & \multirow{2}{*}{\multicolumn{2}{|c|}{$\begin{array}{l}\text { Average score for all three } \\
\text { areas (out of 100) }\end{array}$}} \\
\hline & & & & & & & & & \\
\hline \multirow{2}{*}{ Sex } & Malo & $72.1+20.5$ & 2 & $56.5+26.7$ & 1 & $25.7+28.9$ & 1 & $511+193$ & 1 \\
\hline & Female & $76.0 \pm 14.1$ & 1 & $56.9 \pm 24.2$ & 1 & $24.1 \pm 26.1$ & 1 & $52.3 \pm 21.4$ & 1 \\
\hline \multirow{2}{*}{$\begin{array}{l}\text { Marital } \\
\text { status }\end{array}$} & Married & $75.3 \pm 15.9$ & 1 & $57.3 \pm 24.7$ & 1 & $24.7 \pm 27.0$ & 1 & $52.4 \pm 20.9$ & 1 \\
\hline & Unmarried & $74.2 \pm 15.0$ & 1 & $53.0 \pm 25.0$ & 1 & $22.2 \pm 24.9$ & 1 & $49.8 \pm 21.3$ & 1 \\
\hline \multirow{3}{*}{$\begin{array}{l}\text { Having } \\
\text { children }\end{array}$} & No & $74.9 \pm 15.2$ & 1 & $53.9 \pm 25.2$ & 2 & $25.1 \pm 26.3$ & 1 & $51.3 \pm 20.4$ & 1,2 \\
\hline & One child & $73.3 \pm 16.5$ & 1 & $53.3 \pm 24.7$ & 2 & $23.7 \pm 26.8$ & 1 & $50.1 \pm 20.4$ & 2 \\
\hline & More than a child & $75.7 \pm 15.7$ & 1 & $58.4 \pm 24.5$ & 1 & $24.5 \pm 26.9$ & 1 & $52.9 \pm 21.3$ & 1 \\
\hline \multirow{4}{*}{ Age groups } & $16-25$ & $73.0 \pm 14.8$ & 2 & $51.6 \pm 23.4$ & 3 & $22.2 \pm 24.3$ & 2 & $48.9 \pm 20.8$ & 3 \\
\hline & $26-35$ & $75.4 \pm 16.3$ & 1,2 & $56.6 \pm 24.6$ & 2 & $23.1 \pm 26.8$ & 2 & $51.7 \pm 21.6$ & 2 \\
\hline & $36-45$ & $75.6 \pm 15.4$ & 1,2 & $58.4=24.7$ & 1,2 & $25.4 \pm 26.4$ & 1,2 & $53.1 \pm 20.8$ & 1,2 \\
\hline & $>46$ & $76.5 \pm 16.1$ & 1 & $61.4 \pm 25.8$ & 1 & $28.82 \pm 29.6$ & 1 & $55.4 \pm 20.1$ & 1 \\
\hline \multirow{2}{*}{ Residency } & Madinah City & $75.3 \pm 15.8$ & 1 & $57.3 \pm 24.6$ & 1 & $24.7 \pm 26.9$ & 1 & $52.4 \pm 20.9$ & 1 \\
\hline & Madinah suburb & $73.3 \pm 15.2$ & 1 & $52.3 \pm 26.5$ & 1 & $22.0 \pm 24.8$ & 1 & $49.2 \pm 21.1$ & 2 \\
\hline \multirow{6}{*}{$\begin{array}{l}\text { Educational } \\
\text { level }\end{array}$} & Uneducated & $58.3 \pm 16.0$ & 3 & $42.9 \pm 21.5$ & 2 & $14.3 \pm 29.9$ & 3 & $38.5 \pm 18.2$ & 3 \\
\hline & Elementary & $67.1 \pm 19.1$ & 2 & $38.7 \pm 20.5$ & 2 & $6.7 \pm 11.6$ & 2,3 & $37.5 \pm 24.7$ & 3 \\
\hline & Intermediate & $71.1 \pm 12.7$ & 1,2 & $50.3 \pm 26.8$ & 1,2 & $18.4 \pm 21.5$ & 2,3 & $46.6 \pm 21.7$ & 2 \\
\hline & Secondary & $71.7 \pm 15.8$ & 1,2 & $52.9 \pm 24.3$ & 1,2 & $19.0 \pm 23.7$ & 2,3 & $47.9 \pm 21.8$ & 2 \\
\hline & University & $76.2 \pm 15.3$ & 1,2 & $58.1 \pm 24.6$ & 1 & $25.2 \pm 26.7$ & 2 & $53.2 \pm 21.1$ & 1,2 \\
\hline & Postgraduate & $79.8 \pm 17.9$ & 1 & $63.8 \pm 23.7$ & 1 & $40.0 \pm 31.7$ & 1 & $61.2 \pm 16.4$ & 1 \\
\hline
\end{tabular}

Significance was at $p<0.05$. The difference between means was not significant for subsets of those sharing any common indicator number. The difference was significant in favor of smaller indicator subset numbers 
Table 4: Level of significance of the differences in means of various scores of parents who gave non-prescribed antibiotics to their children

\begin{tabular}{|c|c|c|c|c|c|c|c|c|}
\hline \multirow{2}{*}{$\begin{array}{l}\text { Behavior } \\
(n=400)\end{array}$} & \multicolumn{2}{|c|}{$\begin{array}{c}\text { General knowledge score } \\
\text { (out of 100) }\end{array}$} & \multicolumn{2}{|c|}{$\begin{array}{l}\text { Side effects knowledge score } \\
\text { (out of 100) }\end{array}$} & \multicolumn{2}{|c|}{$\begin{array}{c}\text { Antibacterial resistance } \\
\text { knowledge score (out of } 100 \text { ) }\end{array}$} & \multicolumn{2}{|c|}{ Total score } \\
\hline & Mean $\pm S D$ & $\begin{array}{l}\text { Significance } \\
\text { subsets }\end{array}$ & Mean $\pm S D$ & $\begin{array}{l}\text { Significance } \\
\text { subsets }\end{array}$ & Mean $\pm S D$ & $\begin{array}{l}\text { Significance } \\
\text { subsets }\end{array}$ & Mean $\pm S D$ & $\begin{array}{l}\text { Significance } \\
\text { subsets }\end{array}$ \\
\hline $\begin{array}{l}\text { Buying antibiotics each time the child } \\
\text { was sick }\end{array}$ & & & & & & & & \\
\hline Yes & $61.2 \pm 19.3$ & 2 & $52.5 \pm 23.5$ & 2 & $24.1 \pm 25.0$ & 1 & $51.0 \pm 16.4$ & 2 \\
\hline No & $77.8 \pm 14.5$ & 1 & $59.2 \pm 25.3$ & 1 & $24.5 \pm 25.7$ & 1 & $61.2 \pm 14.3$ & 1 \\
\hline Reason for antibiotic use & & & & & & & & \\
\hline Fever & $69.7 \pm 20.7$ & 1,2 & $54.7 \pm 26.0$ & 1 & $23.8 \pm 26.8$ & 1 & $55.7 \pm 17.6$ & 1,2 \\
\hline Wound & $76.3 \pm 11.6$ & 1 & $60.9 \pm 21.8$ & 1 & $27.7 \pm 23.5$ & 1 & $61.7 \pm 12.9$ & 1 \\
\hline Gastrointestinal symptoms & $68.4 \pm 17.4$ & 2 & $51.6 \pm 25.0$ & 1 & $21.0 \pm 21.5$ & 1 & $53.5 \pm 11.1$ & 2 \\
\hline Cold & $73.1 \pm 16.7$ & 1,2 & $59.2 \pm 23.7$ & 1 & $25.1 \pm 25.2$ & 1 & $59.1 \pm 15.0$ & 1,2 \\
\hline Discontinuation time & & & & & & & & \\
\hline At no symptoms & $63.6 \pm 21.3$ & 2 & $50.5 \pm 24.2$ & 2 & $15.6 \pm 21.4$ & 3 & $49.8 \pm 16.6$ & 2 \\
\hline $\begin{array}{l}\text { After full course of doses without } \\
\text { proper timing }\end{array}$ & $73.1 \pm 16.8$ & 1 & $58.8 \pm 24.3$ & 1 & $22.2 \pm 25.1$ & 2 & $58.3 \pm 14.0$ & 1 \\
\hline $\begin{array}{l}\text { After fuill course of doses with proper } \\
\text { timing }\end{array}$ & $74.6 \pm 16.3$ & 1 & $58.8 \pm 24.7$ & 1 & $29.4 \pm 26.1$ & 1 & $60.5 \pm 15.0$ & 1 \\
\hline $\begin{array}{l}\text { Keeping the remaining doses for future } \\
\text { use }\end{array}$ & & & & & & & & \\
\hline Yes & $59.5 \pm 21.3$ & 2 & $47.8 \pm 24.8$ & 2 & $22.4 \pm 25.1$ & 1. & $48.4 \pm 17.2$ & 2 \\
\hline No & $74.9 \pm 15.9$ & 1 & $59.2 \pm 24.2$ & 1 & $24.9 \pm 25.5$ & 1 & $59.9 \pm 14.5$ & 1 \\
\hline
\end{tabular}

Level of significance was reported at $p<0.05$. The difference between means was not significant for subsets with any common indicator number. The difference was significant in favor of smaller indicator subset numbers 
Participants also indicated various reasons for purchasing antibiotics without consulting a physician or obtaining a prescription. Table 5 shows that two of the major reasons were lack of awareness of the importance of consulting a doctor, and the long waiting durations at clinics before seeing a doctor or obtaining a prescription.

\section{DISCUSSION}

Antibiotic resistance results directly from antibiotic misuse. The greater the volume of antibiotics used, the greater the chances of developing antibiotic-resistant species [19]. Therefore, organized efforts have been jointly made by the healthcare system, users, and prescribers to develop measures to safeguard and sustain antibiotics prescription. Hence, the World Alliance Antibiotic Resistance (WAAR) advocated a number of immediate measurements, which include increased inspection for antibiotic resistance and antibiotic use, with regular feedback to healthcare professionals and the public, and regular education and training programs for healthcare professionals and consumers [20].

The aim of the present study was to assess the level of awareness of proper use of antibiotic among parents in Madinah. Sex, marital status, and residency had very little significant effect on participants' performance in antibiotic-related tests, and hence on their awareness. The number of children participants had, participants' age group, level of education, and the nature of their career seemed to have influenced their overall scores. While these factors did not seem to have greatly influenced the general knowledge score, a clear pattern was observed with the side effects test scores.

Having more than one child or belonging to an older age group increased the side effects test score only slightly, while being more educated clearly influenced the score by at least $20 \%$. Lower scores were observed for the side effects test, when compared to the general knowledge test score. This lower effect was seen with the antibacterial resistance test as well. This indicates that the majority of participants had good basic knowledge of antibiotics use, but very little on antibacterial resistance.

Results from this study clearly suggested that the majority of participants had very limited understanding of what antibacterial resistance was. This could have limited their awareness on antibiotic misuse. In fact, it may be safe to say that a great proportion of Madinah's population considered antibiotics as an over-the-counter medicinal products (OTC), which makes the misuse of antibiotics all the more dangerous [21]. Self-medication with antibiotics may aid in the treatment of minor ailments, and therefore play an important role in decreasing the financial burden due to costs of medical services, especially in countries with limited healthcare resources [22]. However, the disregard for antibiotics prescription regulations is a major cause of irrational antibiotics use and antibiotic resistance.

A study performed in Oman showed that $94 \%$ of participants practice self-medication, while $74 \%$ of parents had a false understanding that antibiotics are always used in the treatment of fever, which is another reason for the antibiotic resistance [22]. In the same study, $16.4 \%$ of parents thought that antibiotics could be used effectively against viruses. Thus, it is very essential to educate parents on the appropriate use of antibiotics appropriately to prevent future diseases among the young through stoppage or at least reduction in the level of antibiotic resistance. The results obtained in this study are similar to results obtained by El Zowalaty and colleagues in Riyadh [19].

Most parents agreed that the lack of awareness was the main reason for obtaining antibiotics without prescriptions. This finding is similar to results obtained from the central region of Saudi Arabia which showed that lack of knowledge and inappropriate beliefs were behind the misuse of antibiotics for children [14]. Moreover, in a study conducted in 2015 to determine the frequency of obesity in children in Madinah, an incidence of 20 $\%$ was found among intermediate male students, with associated high prevalence of Type 1 diabetes mellitus without any obvious cause [17]. Sadly, this was the highest reported incidence in the Middle East. However, no further research has been conducted to investigate the possible causes of the high incidence of Type 1 diabetes [22]. Since these disorders were found in the same region as that surveyed in the present study, a possible link might exist between antibiotic misuse in children and the appearance of Type 1 diabetes mellitus.

\section{Limitations of the study}

The main limitation of this study is that data were gathered from participants from a single city. Despite the fact that Madinah is the capital of its district area, the inclusion of participants from other cities within the district should have given a 
more representative picture of parent's REFERENCES

awareness of antibiotic misuse.

\section{CONCLUSION}

The results from this study indicate that participants showed very limited awareness of antibiotic bacterial resistance. Education is the dominant factor for increasing such awareness. Therefore, awareness campaigns about antibiotics and their side effects are highly recommended for the public. Furthermore, the role of healthcare professionals in educating the population on proper use of antibiotics to ensure better patient compliance must be reinforced. Further studies are needed to determine the correlation between antibiotic misuse and obesity, diabetes, and depression.

\section{DECLARATIONS}

\section{Acknowledgement}

The authors would like to acknowledge Taibah University for providing support and facilities to carry out this study. We would also like to thank all participants for responding to our questionnaire.

\section{Conflict of interest}

No conflict of interest is associated with this work.

\section{Contribution of authors}

We declare that this work was done by the authors named in this article and all liabilities pertaining to claims relating to the content of this article will be borne by them. All authors contributed to data analysis, drafting and revising the article, and gave final approval of the version to be published.

\section{Open Access}

This is an Open Access article that uses a funding model which does not charge readers or their institutions for access and distributed under the terms of the Creative Commons Attribution License (http://creativecommons.org/licenses/by/ 4.0) and the Budapest Open Access Initiative (http://www.budapestopenaccessinitiative.org/rea d), which permit unrestricted use, distribution, and reproduction in any medium, provided the original work is properly credited.
1. Finkelstein JA, Stille C, Nordin J, Davis R, Raebel MA, Roblin $D$, Go AS, Smith D, Johnson CC, Kleinman $K$. Reduction in antibiotic use among US children, 19962000. Pediatrics 2003; 112(3): 620-627.

2. SCHORLING JB, DE SOUZA MA, GUERRANT RL. Patterns of antibiotic use among children in an urban Brazilian slum. Int J Epidemiol 1991; 20(1): 293-299.

3. Kotwani A, Holloway K. Trends in antibiotic use among outpatients in New Delhi, India. BMC Infect Dis 2011; 11(1): 99.

4. Marra F, Patrick DM, Chong M, Bowie WR. Antibiotic use among children in British Columbia, Canada. J Antimicrob Chemother 2006; 58(4): 830-839.

5. Friedman B-C, Schwabe-Warf D, Goldman R. Reducing inappropriate antibiotic use among children with influenza infection. Can Fam Physician 2011; 57(1): 4244.

6. Sabuncu E, David J, Bernède-Bauduin C, Pépin S, Leroy $M$, Boëlle $P-Y$, Watier L, Guillemot $D$. Significant reduction of antibiotic use in the community after a nationwide campaign in France, 2002-2007. PLoS Med 2009; 6(6): e1000084.

7. Panagakou SG, Papaevangelou V, Chadjipanayis $A$, Syrogiannopoulos GA, Theodoridou $M$, Hadjichristodoulou CS. Risk factors of antibiotic misuse for upper respiratory tract infections in children: results from a cross-sectional knowledge-attitude-practice study in Greece. Pediatrics 2012; 2012.

8. Ekwochi U, Chinawa JM, Osuorah CD, Odetunde OI, Obu HA, Agwu S. The use of unprescribed antibiotics in management of upper respiratory tract infection in children in Enugu, South East Nigeria. J Trop Pediatr 2014; 60(3): 249-252.

9. Le TH, Ottosson E, Nguyen TKC, Kim BG, Allebeck P. Drug use and self-medication among children with respiratory illness or diarrhea in a rural district in Vietnam: a qualitative study. J Multidiscip Healthc 2011; 4: 329 .

10. Bi P, Tong S, Parton KA. Family self-medication and antibiotics abuse for children and juveniles in a Chinese city. Soc Sci Med 2000; 50(10): 1445-1450.

11. Agarwal S, Yewale VN, Dharmapalan D. Antibiotics use and misuse in children: a knowledge, attitude and practice survey of parents in India. J Clin Diagn Res 2015; 9(11): SC21.

12. Sa'ed HZ, Taha AA, Araj KF, Abahri IA, Sawalha $A F$, Sweileh WM, Awang $R$, Al-Jabi SW. Parental knowledge, attitudes and practices regarding antibiotic use for acute upper respiratory tract infections in children: a cross-sectional study in Palestine. BMC Pediatr 2015; 15(1): 176.

13. Al-Dossari K. Parental knowledge, attitude and practice on antibiotic use for upper respiratory tract infections in children. Majmaah J Health Sci 2013; 1(1): 39-51.

14. Abobotain AH, Sheerah $H A$, Alotaibi FN, Joury $A U$, Mishiddi RM, Siddiqui AR, Saeed AB. Socio-

Trop J Pharm Res, February 2020; 19(2): 418 
demographic determinants of antibiotic misuse in children. A survey from the central region of Saudi Arabia. Saudi Med J 2013; 34(8): 832-840.

15. Jandhyala SM, Talukdar $R$, Subramanyam $C$, Vuyyuru $H$, Sasikala M, Reddy DN. Role of the normal gut microbiota. World J Gastroenterol 2015; 21(29): 8787.

16. Caricilli $A$, Saad $M$. The role of gut microbiota on insulin resistance. Nutrients 2013; 5(3): 829-851.

17. Sekirov I, Russell SL, Antunes LCM, Finlay BB. Gut microbiota in health and disease. Physiol Rev 2010; 90(3): 859-904.

18. World Medical Association. World Medical Association Declaration of Helsinki: Ethical Principles for Medical Research Involving Human Subjects. JAMA 2013; 310(20): 2191-2194.

19. El Zowalaty ME, Belkina T, Bahashwan SA, El Zowalaty AE, Tebbens JD, Abdel-Salam HA, Khalil Al, Daghriry
SI, Gahtani MA, Madkhaly FM. Knowledge, awareness, and attitudes toward antibiotic use and antimicrobial resistance among Saudi population. Int J Clin Pharm 2016; 38(5): 1261-1268.

20. Carlet J, Rambaud C, Pulcini C. WAAR (World Alliance against Antibiotic Resistance): Safeguarding antibiotics. Antimicrob Resist Infect Control. 2012; 1(1): 25.

21. Al-Dahi AS, Albalawi FA, Al Alwani SS, Al Balawi MM. Prevalence of overweight and obesity among Saudi primary school students in Tabuk, Saudi Arabia 2015. Bangladesh J Med Sci 2016; 15(3): 329-334.

22. Habeb AM, Al-Magamsi MS, Halabi S, Eid IM, Shalaby S, Bakoush O. High incidence of childhood type 1 diabetes in Al-Madinah, North West Saudi Arabia (2004-2009). Pediatr Diabetes 2011; 12(8): 676-681. 